\title{
A One-Week Course of Levofloxacin/Dexamethasone Eye Drops: A Review on a New Approach in Managing Patients After Cataract Surgery
}

\author{
Stanislao Rizzo • Gloria Gambini (D) - Umberto De Vico • \\ Clara Rizzo · Raphael Kilian
}

Received: August 31, 2021 / Accepted: November 17, 2021 / Published online: December 22, 2021

(C) The Author(s) 2021

\begin{abstract}
A new fixed-dose combination of dexamethasone and levofloxacin eye drops has recently been approved for the prevention and treatment of inflammation, and the prevention of infection associated with cataract surgery in adults. This combination has been developed to respond to a series of unmet needs in the practical management of patients undergoing cataract surgery. Namely, despite updated guidelines, many ophthalmologists employ protocols mainly based on their personal experience. As a result, the choice of drugs, treatment duration, and drug association is not
\end{abstract}

S. Rizzo · G. Gambini ( $₫)$ · U. De Vico Ophthalmology Unit, "Fondazione Policlinico Universitario A. Gemelli IRCCS", Largo A. Gemelli 8, Rome, Italy

e-mail: gambini.gloria@gmail.com

S. Rizzo · G. Gambini · U. De Vico

Catholic University Sacro Cuore, Rome, Italy

S. Rizzo

Consiglio Nazionale delle Ricerche, Istituto di

Neuroscienze, Pisa, Italy

C. Rizzo

Ophthalmology, Department of Surgical, Medical and Molecular Pathology and Critical Care

Medicine, University of Pisa, Pisa, Italy

R. Kilian

Ophthalmology Unit, University of Verona, Verona, Italy evidence-based medicine (EBM)-oriented. In addition, antibiotic resistance may occur since antibiotics are used for an extended length of time, even with tapering. Corticosteroids are also prescribed for prolonged periods, frequently without follow-up. Therefore, patient adherence to postsurgical self-care is low, and mainly affects older patients who are the majority undergoing cataract surgery. In both rabbit and human trials, it has been demonstrated that both active ingredients penetrate the ocular system without pharmacokinetic interaction between the two. The concentrations of both ingredients in aqueous humor after their ocular instillation are high enough at the site of action to carry out their expected potent anti-inflammatory and antibiotic activity. Tested in a pivotal study aimed at investigating efficacy and safety of the intended indication, the mixture/compound was noninferior to a 2-week treatment with dexamethasone/tobramycin in preventing or reducing inflammation and in preventing infection when administered for 1 week, followed by the administration of dexamethasone alone for another week. The outcomes obtained by this study suggest that a 1-week course of levofloxacin/dexamethasone eye drops is sufficient to resolve inflammation and prevent infection in patients undergoing cataract surgery. In addition, this study underlines that a follow-up visit after 1 week allows for a decision about whether to stop or continue a treatment in 
patients still experiencing symptoms or inflammation. In conclusion, this new dose combination could represent a turning point in managing patients after cataract surgery, while mostly avoiding antibiotic resistance and improving treatment adherence.

Keywords: Acceptability; Antibiotic resistance; Cataract surgery; Dexamethasone; Inflammation; Levofloxacin; One-week course; Safety

\section{Key Summary Points}

A fixed-dose combination of dexamethasone and levofloxacin eye drops has been approved to prevent and treat inflammation and to prevent infection associated with cataract surgery.

Despite updated guidelines, many ophthalmologists persevere to employ protocols based on personal experience and using non-evidence-based medicine (EBM)-oriented drugs, treatment duration, and associations.

The mixture/compound was non-inferior to a 2-week treatment with dexamethasone/tobramycin to prevent or reduce inflammation and prevent infection, when administered for 1 week, followed by dexamethasone alone for another week.

This new combination could represent a turning point in managing patients after cataract surgery, mostly avoiding antibiotic resistance and improving treatment adherence.

\section{INTRODUCTION}

A new fixed-dose combination (FDC) of levofloxacin and dexamethasone, available as eye drops, has recently obtained European approval for the prevention and treatment of inflammation and the prevention of infection associated with cataract surgery in adults. This product is innovative as it represents the first approved FDC of a quinolone antibiotic and corticosteroid eye drop. Moreover, this novel FDC constitutes an advancement in the development of a new approach in managing patients after cataract surgery. Namely, the levofloxacin/ dexamethasone FDC is proposed as a short-term and straightforward 7-day course immediately after cataract surgery.

The advantages of this FDC depend on the specific components, such as levofloxacin, a broad-spectrum antibiotic effective against many Gram-positive and Gram-negative pathogens, and dexamethasone, the most potent synthetic corticosteroid.

Levofloxacin is a synthetic third-generation fluoroquinolone antibacterial agent that inhibits the supercoiling activity of bacterial DNA gyrase, halting DNA replication [1]. It is the Lisomer of ofloxacin. Levofloxacin preferentially targets DNA gyrase in Gram-negative bacteria and topoisomerase IV in Gram-positive bacteria. Thus, the spectrum of activity against ocular pathogens includes aerobic Gram-positive microorganisms (Staphylococcus aureus MSSA, Streptococcus pyogenes, Streptococcus pneumoniae, viridans group streptococci), aerobic Gramnegative bacteria (E.coli, Haemophilus influenzae, Moraxella catarrhalis, Pseudomonas aeruginosa community isolates), and other organisms (e.g., Chlamydia trachomatis) [1].

Dexamethasone, a synthetic fluorinated corticosteroid $\left(\mathrm{C}_{22} \mathrm{H}_{29} \mathrm{FO}_{5}\right.$; $(11 \beta, 16 \alpha)$-9-fluoro11,17,21-trihydroxy-16-methylpregna-1,4-diene-3,20-dione), is the most potent synthetic analogue of cortisol. Dexamethasone, like all corticosteroids, achieves its anti-inflammatory effects through suppression of vascular endothelial cell adhesion molecules, cyclooxygenase I or II, and through cytokine expression. This action culminates in a reduced expression of pro-inflammatory mediators and the detachment of leukocytes from the vascular endothelium, thereby preventing their migration into inflamed tissue [2]. Dexamethasone, $0.1 \%$ solution, penetrates the aqueous humor sufficiently to exert its potent anti- 
inflammatory activity at the site of action [3]. Dexamethasone is considered the gold standard for managing postoperative ocular inflammation and is the reference treatment for controlled studies on new drugs or treatments in development $[4,5]$.

Therefore, this compound represents an interesting new addition to the FDC eye drop regime, which currently includes two older products: chloramphenicol/betamethasone, launched in 1964, and the combination tobramycin/dexamethasone, launched in 1993 [6]. This new amalgamation has been conceived as an update that can satisfy the requirements of antibiotic effectivity against a broad spectrum of pathogens. Moreover, this new merger can satisfy the need for affective standardized procedures.

This article is based on previously conducted studies and does not contain any new studies with human participants or animals performed by any of the authors.

\section{UNMET NEEDS IN MEDICAL TREATMENT AFTER CATARACT SURGERY}

The origin of the levofloxacin/dexamethasone FDC lies in satisfying some unmet needs arising in the management of patients undergoing cataract surgery. Unfortunately, despite various guidelines that establish practical and standardized procedures, the rate of adoption of these measures in clinical practice remains inappropriately low [7-11]. At present, at least three main clinical issues require precision.

First, the duration of pharmacological treatment after cataract surgery is highly variable; it frequently depends on empirical ground-based personal experience. Moreover, as two main medications are used, such as antibiotics and anti-inflammatory drugs, mostly corticosteroids, the variability of implemented protocols is extreme, considerable, or high.

The most relevant concern is antibiotic resistance $[12,13]$. The resistance rate exceeds $50 \%$ for some antibiotics [14]. Inappropriate antibiotic therapy is the leading cause of this alarming problem [15]. Consequently, prudent stewardship of antibiotics is strongly recommended by the World Health Organization [16].

In addition, the corticosteroid schedule also is highly variable. Indeed, some ophthalmologists prescribe a tapering of corticosteroids eye drops, likewise of systemic corticosteroids. Moreover, as corticosteroids are frequently combined with antibiotics, tapering inevitably prolongs use of antibiotics and promotes antibiotic resistance. Furthermore, adherence to treatment remains a critical aspect in real-life settings, mainly affecting older patients, who are the majority of those undergoing cataract surgery. The simultaneous use of two or more types of eye drops represents relevant barriers for these patients: difficulty in instilling the drops, necessity for multiple administrations at various times of a day, and the setting. Therefore, this new FDC has been developed to satisfy these demanding requirements.

The possibility of using a safe, handy, and effective product may guarantee a high rate of inflammation resolution, good prevention of infections, and optimal adherence. As discussed further, a 1-week course of levofloxacin/dexamethasone has been observed to resolve ocular inflammation and prevent infection in nearly all subjects undergoing cataract surgery. Moreover, the proposal of such a new approach is firmly based on outcomes provided by a sizeable phase 3 trial, outcomes derived by developing an experimental and clinical action plan.

\section{PRECLINICAL STUDIES}

Two main issues have been investigated in the preclinical phase of development: one concerns the microbiological aspects, and the other, the toxicity profile.

The objective of the microbiological study was to evaluate the in vitro antibacterial activity of the levofloxacin/dexamethasone combination against the pathogens most commonly responsible for external ocular infections and postsurgical endophthalmitis. One aim was to demonstrate that dexamethasone does not interfere with the antibacterial activity of levofloxacin. To this end, the spectrum of activity of the FDC, dexamethasone alone, and 
levofloxacin alone was in vitro investigated to identify Gram-positive and Gram-negative isolates (data on file). Strains that were tested include methicillin-susceptible S. aureus (MSSA), methicillin-resistant S. aureus (MRSA), methicillin-susceptible Staphylococcus epidermidis (MSSE), methicillin-resistant Staphylococcus epidermidis (MRSE), group A S.pyogenes, $S$. pneumoniae, beta-lactamase positive and negative strains of $H$. influenzae, M. catarrhalis, Enterobacter cloacae, and P. aeruginosa. These pathogenic microorganisms were isolated from hospitalized patients with respiratory infections.

The broth cultures of the single strains were diluted in sterile saline solution to obtain a final concentration of $5 \times 10^{5} \mathrm{CFU} / \mathrm{mL}$. Different concentrations of FDC, levofloxacin, and dexamethasone were tested. Finally, the minimum inhibiting concentrations (MIC) and minimum bactericidal concentrations (MBC) were calculated.

The results demonstrated that the FDC was effective as levofloxacin alone against the pathogens evaluated (with the sole exception of some strains of MRSE) by exerting bactericidal activity and with essentially analogous MIC50 and MIC90 values [17]. Therefore, the results of this in vitro study indicated that, from a microbiological perspective, dexamethasone does not affect the antimicrobial activity of levofloxacin.

The second issue concerned the assessment of ocular toxicity. For this purpose, an animal study was performed (data on file). Levofloxacin/dexamethasone eye drop solution was administered to male and female New Zealand White rabbits for four consecutive weeks as a 4-a-day ( $2 \mathrm{~h}$ apart) eye instillation into the conjunctival sac of the right eye at different concentrations and volumes. General clinical observations and eye clinical observations were performed twice daily before the first and the third daily dosing. The results demonstrated that a 4-week, 4-a-day ( $2 \mathrm{~h}$ apart), eye instillation into the conjunctival sac of the right eye of different concentrations of the eye drop combination did not induce any local reaction, even in the presence of a good distribution of the tested drugs in eye matrices. The interpretation of the toxicokinetic data evidenced that the active substances levofloxacin and dexamethasone reached high concentrations at the site of the intended pharmacological activity, namely the ocular tissues, and low concentrations in plasma.

Systemically, there were findings on kidneys and liver in all dose groups, and findings attributable to an exaggerated effect of dexamethasone on adrenals, spleen, thymus, and lymph nodes in all dose groups. Therefore, on the basis of these findings, a no observed adverse effect level could be established for the eye drop combination at the concentration of levofloxacin/dexamethasone $5.12+1.32 \mathrm{mg} /$ $\mathrm{mL}$, given as $30 \mu \mathrm{L} /$ instillation four times a day, which corresponds to the dose proposed for clinical use.

\section{HUMAN STUDIES}

Two human studies have been conducted on the FDC levofloxacin/dexamethasone.

The first study aimed to demonstrate the absence of pharmacokinetic interference between the two active ingredients present in the eye drop combination (Clinical Study Report, data on file). Therefore, the objective of this study was to compare the penetration of levofloxacin and dexamethasone into the aqueous humor (AH) after topical ocular administration in combination and as single active ingredients. The study was randomized, assessor-blinded, and with parallel groups.

A total of 125 patients were randomized: 42 to the levofloxacin + dexamethasone eye drops arm (L-D), 42 to the levofloxacin eye drops arm (L), and 41 to the dexamethasone eye drops arm (D). Patients were on average 74 years of age, $55.20 \%$ were female, and all but three $(97.60 \%)$ were Caucasian. No clinically relevant differences in demographic and background characteristics were found between groups.

The results of the aqueous humor analysis showed that no notable differences in the aqueous humor concentration of levofloxacin were found between the L-D arm and the levofloxacin arm. The mean concentration of levofloxacin was $0.71 \mu \mathrm{g} / \mathrm{mL}$ in the L-D group and 
$0.77 \mu \mathrm{g} / \mathrm{mL}$ in the levofloxacin group. Thus, the concentrations of levofloxacin in the aqueous humor are above the MICs for most of the ocular pathogens in levofloxacin's spectrum of activity. Also, the aqueous humor concentrations of dexamethasone were slightly lower in the levofloxacin + dexamethasone combination arm than in the single-agent $\mathrm{D}$ arm and equal to $11.77 \mathrm{ng} / \mathrm{mL}$ in the L-D group and $16.48 \mathrm{ng} / \mathrm{mL}$ in the D group. In addition, dexamethasone sodium phosphate was not detected in the HA samples of all patients, suggesting its complete hydrolysis to free dexamethasone.

Only one treatment-emergent adverse event was reported: mild mydriasis in the dexamethasone sodium phosphate arm.

In conclusion, this study excluded any pharmacokinetics interference between levofloxacin and dexamethasone related to transcorneal absorption, ensuring AH concentrations of levofloxacin higher than the MIC for the primary pathogens.

The second study aimed to demonstrate the clinical efficacy and safety of the FDC. For this purpose, an international study compared the FDC of levofloxacin and dexamethasone eye drops with the FDC of dexamethasone and tobramycin eye drops [18]. The study was conducted according to good clinical practice procedures. The design was multicenter, randomized, blinded-assessor, parallel-group. The trial evaluated the non-inferiority of 1-week levofloxacin/dexamethasone eye drops (L-D), followed by 1-week dexamethasone alone, with 2-week tobramycin/dexamethasone (one drop QID for all schedules). The aims were to prevent and treat ocular inflammation and prevent infection after uncomplicated cataract surgery. Non-inferiority was defined as the lower limit of the 95\% confidence interval (CI) around a treatment difference greater than $-10 \%$. The study randomized 808 patients to one of two treatment groups in a 1:1 ratio; the randomization was stratified by center. Treatment began immediately after surgery (day 0 ) or after the removal of the eye dressing. Control visits were performed after 3,7 , and 14 days of therapy to evaluate efficacy, compliance, safety, and tolerability.
The test eye drops (L-DSP) were prescribed for 7 days, followed by dexamethasone alone $\left(\right.$ Maxidex $^{\circledR}$ ) for an additional 7 days. The control arm was dexamethasone and tobramycin $\left(\right.$ Tobradex $^{\circledR}$ ) for 14 days. For all eye drops, the dosage was one drop, four times a day.

The physician in charge of assessing study parameters was blinded to treatment assignment. A separate investigational staff member was unblinded and responsible for assigning and dispensing/returning study products.

The primary endpoint was the proportion of patients without anterior chamber inflammation (sum of cells and flare score $=0$ ) assessed by slit-lamp examination after the end of treatment. Endophthalmitis was the key secondary endpoint.

The two groups of patients were homogeneous concerning baseline characteristics.

After the treatment, $95.2 \%$ of the patients in the test arm versus $94.9 \%$ of the control arm had no signs of inflammation in the anterior chamber (difference between proportions of patients $=0.028$ ), as reported in Fig. 1 . However, more than $85 \%$ of patients had no inflammation in the anterior chamber just after the first week of treatment. It has to be noted that about the $90 \%$ of the patients with remaining inflammatory cells in the anterior chamber had a mild inflammation grade, such as 1-5 cells alone and aqueous flare (Tyndal effect) as a barely detectable trace. Only 1\% of patients showed moderate inflammation, such as $6-15$ cells and aqueous flare of mild intensity, after 1 week, but no subject had severe inflammation after 1 week of levofloxacin/dexamethasone. Consistently, conjunctival hyperemia significantly disappeared in most patients in the first week, without an intergroup difference as reported in Fig. 2. In addition, more than 80\% of patients had no symptoms after the first week, without a significant intergroup difference (Fig. 3).

Thus, the non-inferiority of the new short pharmacological strategy was proved. One week of dexamethasone and levofloxacin ensured complete control of inflammation in almost all patients. No case of endophthalmitis was reported. No statistically significant difference was evident in any of the other secondary 


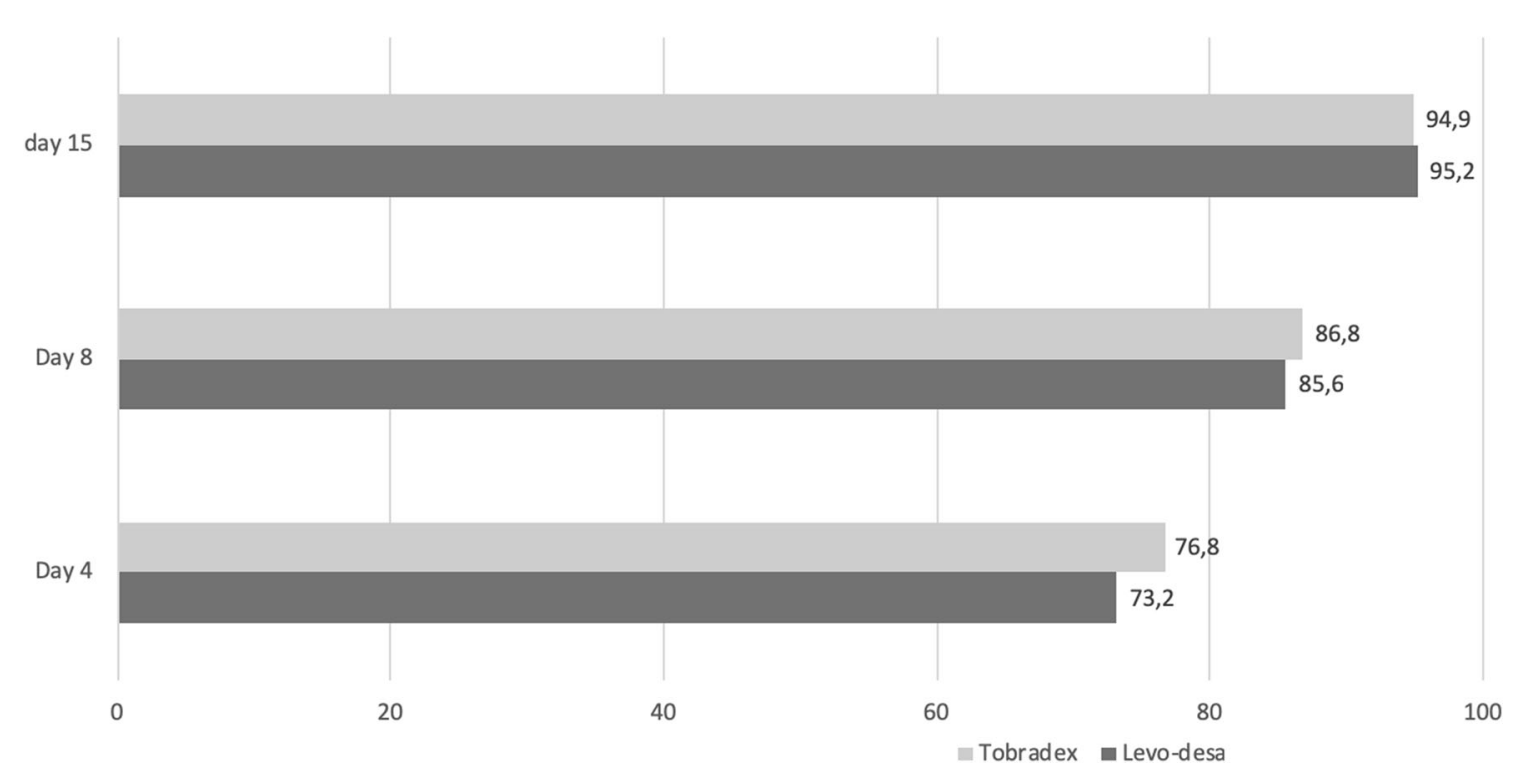

Fig. 1 Percentages of patients without inflammation in the anterior chamber in the two groups at day 4, day 8 , and day 15 . Dark box = Levo-desa group; light box $=$ Tobradex group

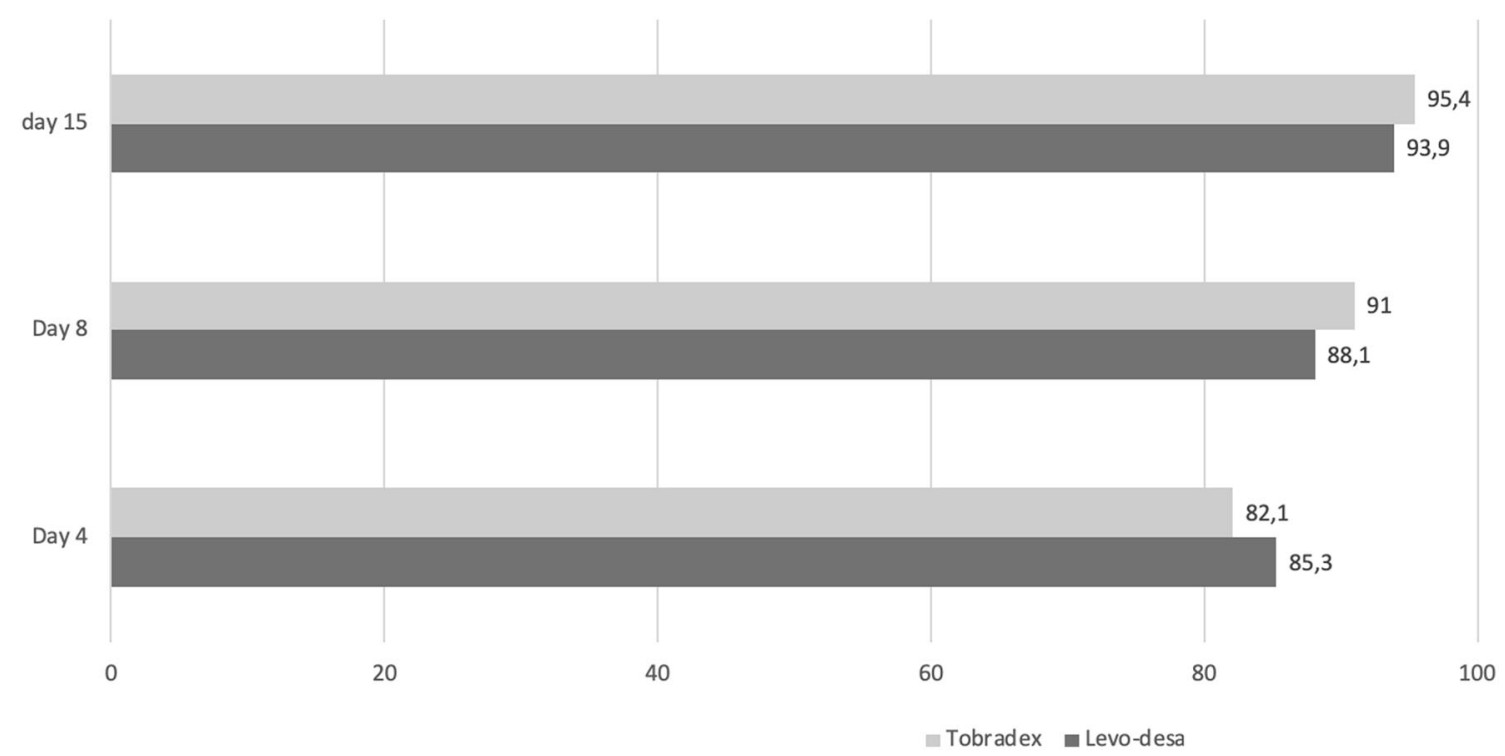

Fig. 2 Percentages of patients without conjunctival hyperemia in the two groups at day 4, day 8, and day 15. Dark box $=$ Levo-desa group; light box $=$ Tobradex group

endpoints. The result was supported by the consistency of the sensitivity and per protocol (PP) analyses. Therefore, the study met its primary objective. Both treatments were well tolerated.

A review of the side effects of the FDC dexamethasone and levofloxacin eye drops included the safety data reported by the 438 patients who effectively took the treatment and underwent cataract surgery.

In the absorption study, single doses of the FDC dexamethasone and levofloxacin were considered well tolerated, and no adverse events were reported.

In the efficacy study, at least one treatmentemergent adverse event (TEAE) was reported for 


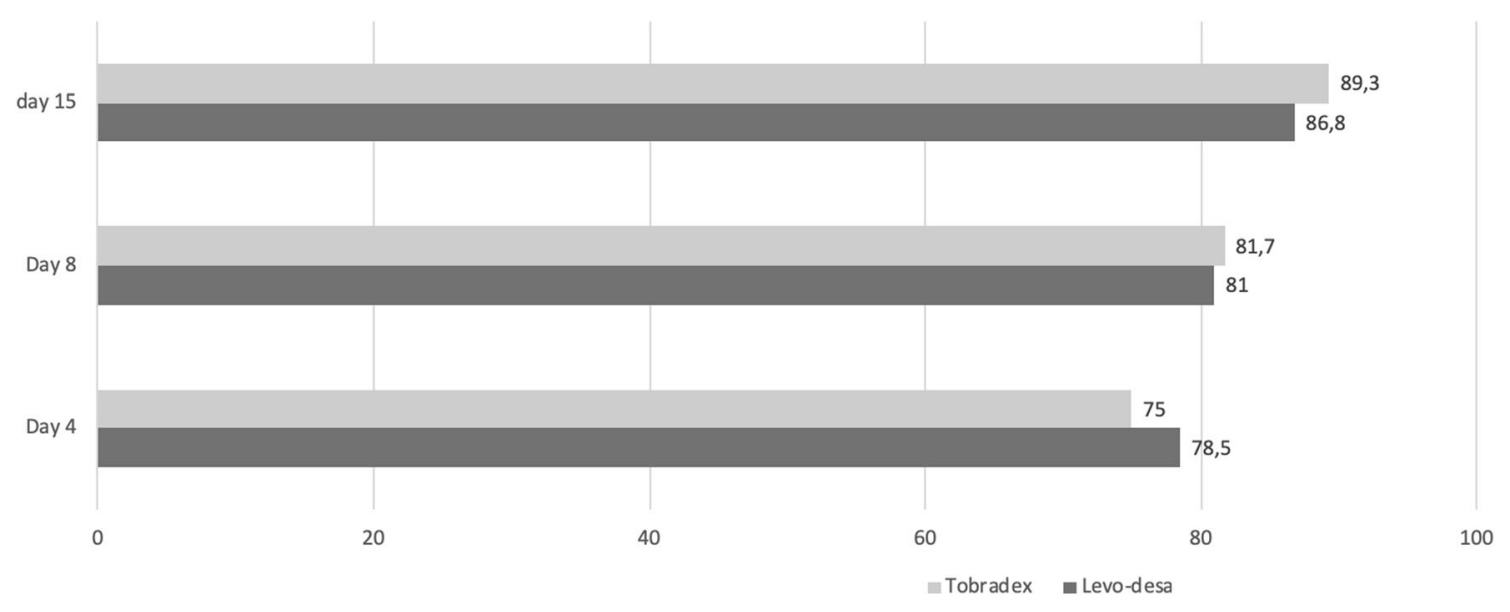

Fig. 3 Percentages of patients without ocular symptoms in the two groups at day 4, day 8, and day 15. Dark box $=$ Levodesa group; light box $=$ Tobradex group

107 of the 788 patients of the safety set, $14.18 \%$ in the FDC dexamethasone levofloxacin followed by dexamethasone alone group, and $12.98 \%$ in the dexamethasone and tobramycin group. No serious adverse event was related to the study treatment. Ocular TEAEs of the operated eye were reported for $8.86 \%$ of the FDC dexamethasone and levofloxacin followed by dexamethasone alone arm, and $10.43 \%$ of the dexamethasone and tobramycin arm. No substantial difference in the distribution of ocular events was present. Corneal edema was the most common TEAE and was reported in 3.29\% of the FDC dexamethasone and levofloxacin arm, and $4.83 \%$ of the dexamethasone and tobramycin arm. Most of those ocular AEs (including corneal edema) in both groups were likely due to phacoemulsification rather than treatment. A significant increase from baseline in intraocular pressure was defined as one in excess of $6 \mathrm{mmHg}$. The proportions of patients with a significant increase after 3,7 , and 14 days of treatment were $1.27 \%$ vs. $1.27 \%, 0.51 \%$ vs. $1.78 \%$, and $1.03 \%$ vs. $0.51 \%$ in the FDC dexamethasone and levofloxacin followed by dexamethasone alone, and in dexamethasone and tobramycin groups, respectively (differences not significant).

Overall, it was concluded that adverse events reported using the FDC dexamethasone and levofloxacin were mild and well tolerated. The most common adverse events were related to ocular discomfort, which could be due to cataract surgery. Furthermore, no related serious adverse events or deaths were reported in clinical studies, confirming the good safety profile of the FDC dexamethasone and levofloxacin.

\section{PRACTICAL ASPECTS}

On the basis of these preclinical and clinical studies, the FDC levofloxacin/dexamethasone eye drops were approved in Europe. The approved indication is prevention and treatment of inflammation and prevention of infection associated with cataract surgery in adults. The approved dosage is one drop instilled into the conjunctival sac after surgery every $6 \mathrm{~h}$. The duration of treatment is 7 days. Care should be taken not to discontinue therapy prematurely. If one dose is missed, treatment should continue with the next dose as planned.

Re-evaluation of the patient to assess the need to continue administering corticosteroid eye drops as monotherapy is recommended after completing 1 week of therapy with dexamethasone and levofloxacin eye drops. The length of this treatment can depend on the patient's risk factors and outcome of surgery and must be determined by the doctor according to slit-lamp microscopic findings and depending on the severity of the clinical picture. 
Follow-up treatment with steroid eye drops should not usually exceed 2 weeks. However, care should be taken not to discontinue therapy prematurely.

The product is not approved in patients of pediatric age; no dose adjustment in the elderly is necessary, but care should be taken in treating patients with renal or liver insufficiency.

Moreover, the outcomes provided by that pivotal trial may suggest two other primary practical considerations. First, a short antibiotic course, such as 1 week, may indeed contribute to limiting the incidence of new antibiotic resistance. In this regard, a very recent narrative review underlined the main factors involved in antibiotic resistance [19]. Second, widespread inappropriate use of antibiotics has emerged as one of the leading causes of antibiotic resistance, whose adverse outcomes call for the development of antibiotic stewardship programs and global surveillance networks. Misuse, here, refers to high consumption of antibiotics, often unnecessarily, and it comprises overuse, inappropriate prescribing, self-medication, negligent use, or incorrect dosage or treatment duration.

Moreover, as cataract surgery is the most common surgery in western countries, it is obvious how this problem may be compelling, especially since a good clinical practice based on prudent antibiotic stewardship is welcome.

Furthermore, an effective and safe 1-week course using an FDC may significantly improve the adherence of patients. For example, a recent study highlighted the negative impact of multiple prescriptions in patients undergoing cataract surgery [20]. In addition, the use of different eye drops, frequently associated with several concomitant medications for comorbidities, is associated with poor adherence to prescribed eye drop regimens [21]. As a result, the prescription of a single FDC course for only 1 week may undoubtedly improve compliance and accommodate patient preference.

\section{CONCLUSION}

This FDC levofloxacin/dexamethasone product represents the first combination of a quinolone antibiotic with a corticosteroid in eye drops at the dosage of $4 \times 30-\mu \mathrm{L}$ drops for 7 days.

This product has been tested in a pivotal study conducted on adult and elderly patients undergoing cataract surgery to prevent and treat inflammation, and prevent infection associated with cataract surgery [18]. The FDC levofloxacin/dexamethasone, administered for 1 week, followed by the administration of dexamethasone alone for another week, was noninferior to a 2 -week treatment with a combination of dexamethasone and tobramycin in preventing or reducing inflammation and in preventing infection. Indeed, these outcomes represent the primary goals of combined therapy in ophthalmic surgery. Furthermore, efficacy and safety results were comparable between the two treatment groups for all endpoints. Remarkably, $99 \%$ of patients had a complete resolution of inflammatory signs or very mild signs of inflammation (1-5 cells in the aqueous humor and flare as a barely detectable trace) already after the first 7 days of treatment in both groups of patients. Consistently, symptoms were absent or very minimal in these subjects. With regard to the possible side effects of topical ocular treatment with corticosteroids, this finding may lead to a reevaluation of the duration of corticosteroid treatment following cataract surgery to further improve the risk/benefit ratio of the treatment.

From a clinical point of view, of particular importance is that prevention of infection was similarly effective in patients treated with FDC dexamethasone and levofloxacin for only 7 days compared to the control arm of the antibiotic treatment administered for 14 days. Considering that the duration of antibiotic administration is one of the main factors facilitating the appearance of bacterial resistance, the results of this study, which support the efficacy and safety of short antibiotic prophylaxis, can contribute significantly to the policy of containment of the use of antibiotics [22-24]. Indeed, antimicrobial resistance is a global problem, and ocular pathogens are no exception [25]. Therefore, many health authorities and medical associations have advocated antibiotic stewardship. It has been defined in a consensus statement from the Infectious 
Diseases Society of America, the Society for Healthcare Epidemiology of America, and the Pediatric Infectious Diseases Society [26]. Antibiotic stewardship includes "coordinated interventions designed to improve and measure the appropriate use of antibiotic agents by promoting the selection of the optimal antibiotic drug regimen including dosing, duration of therapy, and route of administration." The benefits of antibiotic stewardship include improved patient outcomes, reduced adverse events, improvement in rates of antibiotic susceptibilities to antibiotics, and optimization of resource utilization across the continuum of care. The European Society of Cataract and Refractive Surgeons (ESCRS) guidelines do not indicate a specific antibiotic for topical postoperative treatment or a precise duration of such treatment. However, they emphasize the importance of rational use to limit bacterial resistance development, mainly when a broadspectrum antibiotic is used [8]. Among the most widely used FDCs in eye drop formulations is tobramycin and dexamethasone, whose administration usually lasts for at least 2 weeks, sometimes tapering posology over several weeks. Tapering is, however, inappropriate in preventing the development of bacterial resistance, which is favored by prolonged treatment and the use of sub-therapeutic doses. For this reason, antibiotic treatment should not be prolonged beyond the time necessary for the healing of surgically induced epithelial lesions, and therefore should not last more than a week [27].

The efficacy study by Bandello et al. [18] proved that the use of the combination of levofloxacin and dexamethasone sodium phosphate for a limited time of 1-week, followed by treatment with a corticosteroid alone, is noninferior in reducing and preventing postoperative inflammation and the incidence of postoperative infections when compared to 2-week treatment using a combination of dexamethasone and tobramycin. Therefore, the FDC dexamethasone and levofloxacin administered with a shortened regimen may represent an opportunity to optimize the use of antimicrobials in ophthalmic surgery.
All treatments were found to be safe. Among the adverse events defined as "possibly related" to treatment with either the test or the reference drug regimen, the following ocular events were likely due to phacoemulsification rather than to the drug treatment: corneal edema, eye irritation, abnormal sensation in the eye, increased lacrimation, asthenopia, corneal disorder, dry eye, eye pain, ocular discomfort, uveitis, visual brightness, and conjunctivitis.

FDC eye drops are comfortable for patients and improve medication compliance. This aspect is of great importance in elderly persons, representing at least $80 \%$ of the target population of dexamethasone and levofloxacin FDC eye drops [28]. Moreover, FDCs are superior to the extemporaneous combination of different products, as the FDCs are guaranteed by Chemistry, Manufacturing, and Controls (CMC) investigation and preclinical and clinical studies [29-31]. Therefore, the efficacy and safety in humans and the non-clinical toxicity profile and safety margin for human use are carefully investigated for FDCs. On the contrary, extemporaneous combinations are mainly based on empirical use, and their risk for patients may be underestimated. In addition, the use of an FDC investigated in a well-designed absorption study would avoid the dilution in the cul-de-sac of empirically combined active ingredients and a possible consequent decrease of efficacy of either product.

In conclusion, the therapeutical needs, such as the prompt resolution of inflammation and prevention of infections, could be safely answered by this new approach based on a short course of FDC with levofloxacin and dexamethasone. This new proposal could pave the way to more rational and safe management of patients undergoing cataract surgery, mainly in elderly subjects.

\section{ACKNOWLEDGEMENTS}

Funding. No funding or sponsorship was received for this study. The journal's Rapid 
Service Fee was funded by NTC S.r.l, MilanItaly

Authorship. All named authors meet the International Committee of Medical Journal Editors (ICMJE) criteria for authorship for this article, take responsibility for the integrity of the work as a whole, and have given their approval for this version to be published.

Author Contributions. Conceptualization: Stanislao Rizzo, writing the manuscript: Gloria Gambini, writing the manuscript: Umberto De Vico, manuscript revision and conceptualization: Clara Rizzo, manuscript revision and design: Raphael Kilian.

Disclosures. Stanislao Rizzo, Gloria Gambini, Umberto De Vico, Clara Rizzo and Rapahel Kilian all have nothing to disclose.

Compliance with Ethics Guidelines. This article is based on previously conducted studies and does not contain any new studies with human participants or animals performed by any of the authors.

Data Availability. Data sharing is not applicable to this article as no datasets were generated or analyzed during the current study.

Open Access. This article is licensed under a Creative Commons Attribution-NonCommercial 4.0 International License, which permits any non-commercial use, sharing, adaptation, distribution and reproduction in any medium or format, as long as you give appropriate credit to the original author(s) and the source, provide a link to the Creative Commons licence, and indicate if changes were made. The images or other third party material in this article are included in the article's Creative Commons licence, unless indicated otherwise in a credit line to the material. If material is not included in the article's Creative Commons licence and your intended use is not permitted by statutory regulation or exceeds the permitted use, you will need to obtain permission directly from the copyright holder. To view a copy of this licence, visit http://creativecommons.org/licenses/bync/4.0/.

\section{REFERENCES}

1. Anderson VR, Perry CM. Levofloxacin: a review of its use as a high-dose, short-course treatment for bacterial infection. Drugs. 2008;68(4):535-65.

2. Holland EJ, Fingeret M, Mah FS. Use of topical steroids in conjunctivitis: a review of the evidence. Cornea. 2019;38(8):1062-7.

3. Mogensen TH, Berg RS, Paludan SR, Ostergaard L. Mechanisms of dexamethasone-mediated inhibition of Toll-like receptor signaling induced by Neisseria meningitidis and Streptococcus pneumoniae. Infect Immun. 2008;76:189-97.

4. Abadia B, Calvo P, Ferreras A, Bartol F, Verdes G, Pablo L. Clinical applications of dezamethasone for aged eyes. Drugs Aging. 2016;33:639-46.

5. Grzybowski A, Brockmann T, Kanclerz P, Pleyer U. Dexamethasone intraocular suspension. A longacting therapeutic for treating inflammation associated with cataract surgery. J Ocul Pharmacol Ther. 2019;35:525-34.

6. Camesasca FI, Bianhi C, Beltrame G, et al. Control of inflammation and prophylaxis of endophthalmitis after cataract surgery: a multicenter study. Eur J Ophthalmol. 2007;17:733-42.

7. Ong-Tone L, Bell A, Tan YY. Practice patterns of Canadian Ophthalmological Society members in cataract surgery: 2011 survey. Can J Ophthalmol. 2012;47:124-30.

8. Barry P, Cordovés L, Gardner S. ESCRS guidelines for prevention and treatment of endophthalmitis following cataract surgery: data, dilemmas and conclusions. 2013. www.escrs.org.

9. Wu CM, Wu AM, Young BK, et al. An evaluation of cataract surgery clinical practice guidelines. Br J Ophthalmol. 2015;99:401-4.

10. ESCRS Endophthalmitis Study Group. Prophylaxis of postoperative endophthalmitis following cataract surgery: results of the ESCRS multicenter study and identification of risk factors. J Cataract Refract Surg. 2007;33(6):978-88.

11. Montan PG, Wejde G, Setterquist H, Rylander M, Zetterström C. Prophylactic intracameral cefuroxime. Evaluation of safety and kinetics in cataract surgery. J Cataract Refract Surg. 2002;28(6):982-7. 
12. Alós JI. Antibioticv resistance: a global crisis. Enferm Infecc Microbiol Clin. 2015;33(10):692-9.

13. Almakki A, Jumas-Bilak E, Marchandin H, LicznarFajardo P. Antibiotic resistance in urban runoff. Sci Total Environ. 2019;1(667):64-76.

14. Chen J, Li W, Zhang J, et al. Prevalence of antibiotic resistance genes in drinking water and biofilms: the correlation with the microbial community and opportunistic pathogens. Chemosphere. 2020;259: 127483.

15. Guo H, Hildon ZJ, Loh VWK, et al. Exploring antibiotic prescribing in public and private primary care settings in Singapore: a qualitative analysis informing theory and evidence-based planning for value-driven intervention design. BMC Fam Pract. 2021;22(1):205.

16. WHO. Worldwide country situation analysis: response to antimicrobial resistance. https://www. who.int/drugresistance/documents/ situationanalysis/en.

17. Matsuura K, Miyazaki D, Sasaki SI, Inoue Y, Sasaki Y, Shimizu Y. Conjunctival bacterial flora and antimicrobial susceptibility in bacterial pathogens isolated prior to cataract surgery. Jpn J Ophthalmol. 2020;64(4):423-8.

18. Bandello F, Coassin M, Di Zazzo A, et al. One week of levofloxacin plus dexamethasone eye drops for cataract surgery: an innovative and rational therapeutic strategy. Eye (Lond). 2020;34(11):2112-22.

19. Giacomini E, Perrone V, Alessandrini D, Papi D, Nappi C, Degli EL. Evidence of antibiotic resistance from population-based studies: a narrative review. Infect Drug Resist. 2021;14:849-58.

20. Matossian C. Noncompliance with prescribed eyedrop regimens among patients undergoing cataract surgery-prevalence, consequences, and solutions. US Ophthalm Rev. 2020;13:18-22.

21. Lai $\mathrm{Y}, \mathrm{Wu} \mathrm{Y}$, Chai $\mathrm{C}$, et al. The effect of patient education and telemedicine reminders on adherence to eye drops for glaucoma. Ophthalmol Glaucoma. 2020;3:369-76.

22. Pilatz A, Dimitropoulos K, Veeratterapillay R, et al. Antibiotic prophylaxis for the prevention of infectious complications following prostate biopsy: a systematic review and meta-analysis. J Urol. 2020;204(2):224-30.
23. Komolafe O, Roberts D, Freeman SC, et al. Antibiotic prophylaxis to prevent spontaneous bacterial peritonitis in people with liver cirrhosis: a network meta-analysis. Cochrane Database Syst Rev. 2020;1(1):CD013125.

24. Ceraudo M, Prior A, Balestrino A, et al. Ultra-short antibiotic prophylaxis guided by preoperative microbiological nasal swabs in endoscopic endonasal skull base surgery. Acta Neurochir (Wien). 2021;163(2):369-82.

25. Asbell PA, Sanfilippo CM, Sahm DF, DeCory HH. Trends in antibiotic resistance among ocular microorganisms in the United States from 2009 to 2018. JAMA Ophthalmol. 2020;138(5):439-50.

26. Barlam TF, Cosgrove SE, Abbo LM, et al. Implementing an antibiotic stewardship program: guidelines by the Infectious Diseases Society of America and the Society for Healthcare Epidemiology of America. Clin Infect Dis. 2016;62(10):e51-77.

27. Porela-Tiihonen S, Kokki H, Kaarniranta K, Kokki M. Recovery after cataract surgery. Acta Ophthalmol. 2016;94(Suppl 2):1-34.

28. Lee MD, Chen SP, Chen TA, et al. Characteristics of cataract surgery patients influencing patient satisfaction scores. J Cataract Refract Surg. 2019;45(4): 437-42.

29. Barraquer RI, de Toledo JPA, Montané D, Escoto RM, Torres CG, Bennani-Tazzi M. Fixed-dose combination of $0.1 \%$ diclofenac plus $0.3 \%$ tobramycin ophthalmic solution for inflammation after cataract surgery: a randomized, comparative, active treatment-controlled trial. Eur J Ophthalmol. 1998;8(3):173-8.

30. Ornek K, Yalçindağ FN, Ozdemir O. Corneal melting associated with a fixed-dose combination of diclofenac $0.1 \%$ plus tobramycin $0.3 \%$ following cataract surgery. J Cataract Refract Surg. 2008;34(8): 1417.

31. Cunha PA, Shinzato FA, Tecchio GT, Weber SL, Brasil A, Avakian A. Efficacy and tolerability of a gatifloxacin/prednisolone acetate fixed combination for topical prophylaxis and control of inflammation in phacoemulsification: a 20-day-doubleblind comparison to its individual components. Clinics (Sao Paulo). 2013;68(6):834-9. 